EESTI NSV TEADUSTE AKADEEMIA TOIMETISED. XV KOIDE FOUSIKA-MATEMAATIKA- JA TEHNIKATEADUSTE SEERIA. 1966, NR. 4

ИЗВЕСТИЯ АКАДЕМИИ НАУК ЭСТОНСКОИ ССР. ТОМ ХV

СЕРИЯ ФИЗИКО-МАТЕМАТИЧЕСКИХ И ТЕХНИЧЕСКИХ НАУК, 1966, № 4

\title{
P. ЛЭЭHE
}

\section{О НЕРАВНОМЕРНОСТИ ВРЕМЕНИ ПРЕБЫВАНИЯ ЧАСТИЦ В КИПЯЩЕМ СЛОЕ}

Ряд исследователей $[1-5]$ независимо друг от друга определили экспоненциальную зависимость распределения времени пребывания частиц в реакторе с кипящим слоем непрерывного действия, исходя из предположекия, что реакторы работают как идеальные смесители.

В работах $[3-5]$ она была представлена в вияде

$$
\varphi(\tau)=\frac{1}{\tau_{c p}} \exp \left(-\frac{\tau}{\tau_{c p}}\right)
$$

где $\varphi(\tau)$ - плотность вероятности для выхода материала; $\tau$ и $\tau_{\mathrm{cp}}$ - текущее и среднерасходное время пребывания в слое.

При математическом описании вышеуказанные авторы пренебрегли -минимальнымвременем, потребным для прохождения частицей от места входа к месту выхода без всяких столкновений.

В промышленных, крупногабаритных реакторах, особенно при перекрестном движении псевдоожижающего агента и материана,- минимальное возможное время пребывағия частиц в реакторе может значительно отличаться от нуля. Ниже выводится уравнение плотности вероятности для выхода материала с учетом влияния минимального времени. $\tau_{\text {миня }}$

Время выдержки частиц в кипящем слое можно считать непрерывной случайной величиной $\tau^{*}$. Тогда неравномернасть времени выдержки определена интегралььным законом распределения $F(\tau)$ и дифференциаль: ным законом распределения $\varphi(\tau)$. Предполагаем, что вероятность $P$ выхода частицы из кипящего слоя за небольшой промежуток времени наблюдения приблизительно пропорциональна этому промежутку времени и не зависит от времени нахождения частицы в слое до этого.

Пусть событие $A$ состоит в том, что частица находится в слое не менее $\tau$ секунд, а событне $B-$ в том, что частица выходит из реактора между $\tau$ и $\tau+\Delta \tau$ секунд.

Мы имеем:

$$
\begin{aligned}
& P(A)=P\left(\tau^{*} \geqslant \tau\right)=1-P\left(\tau^{*}<\tau\right)=1-F(\tau) \\
& P(B)=P\left(\tau \leqslant \tau^{*}<\tau+\Delta \tau\right)=F(\tau+\Delta \tau)-F(\tau) .
\end{aligned}
$$

Так как мы наблюдаем за частицами, находящимися в слое, то определяется не сама вероятность $P(B)$, а условная вероятность $P_{A}(B)$. 
Заметим, что если событие $B$ произошло, то наверняка произошло и событие $A$, т. е. $P_{B}(A)=1$. Из теоремы умножения

$$
P(A) P_{A}(B)=P(B) P_{B}(A)
$$

находим, что

$$
P_{A}(B)=\frac{P(B)}{P(A)}=\frac{F(\tau+\Delta \tau)-F(\tau)}{1-F(\tau)}
$$

Если $\Delta \tau$ достаточно мало, то можно записать, что

$$
P_{A}(B) \approx \frac{\varphi(\tau) \Delta \tau}{1-F(\tau)}
$$

где $\varphi(\tau)=F^{\prime}(\tau)$ - дифференциальный закон распределения. C другой стороны, по условию вероятность $P_{A}(B)$ приближенно равна

$$
P_{A}(B) \approx k \Delta \tau,
$$

где $k$ - коэффициент пропорциональности.

Сравнивая приближенные равенства (2) и (3) и учитывая, что ошибка их стремится к нулю при $\Delta \tau \rightarrow 0$, получаем

$$
\varphi(\tau)=k-k F(\tau)
$$

Дифференцируя последнее равенство, приходим к дифференциальному уравнению для искомой плотности вероятности

$$
\varphi^{\prime}(\tau)=-k \varphi(\tau) \text {. }
$$

Интегрирование этого уравнения дает

$$
\varphi(\tau)=C \exp (-k \tau)
$$

Из свойств дифференциального закона распределения имеем

$$
\int_{-\infty}^{\infty} \varphi(\tau) d \tau=1
$$

Для нахождения постоянной $C$ заметим, что в данном случае $\tau^{*}>\tau_{\text {мин, }}$, поэтому для $\tau<\tau_{\text {мвн }}$ имеем $\varphi(\tau) \equiv 0$ и

$$
\int_{\tau_{\text {мин }}}^{\infty} C \exp (-k \tau) d \tau=\frac{C}{k} \exp \left(-k \tau_{\text {мин }}\right)=1 \text {, }
$$

T. e.

$$
C=k \exp \left(k \tau_{\text {мин }}\right) \text {. }
$$

Среднее время пребывания частиц в реакторе определено уравнснием

$$
\tau_{\mathrm{cp}}=\int_{\tau_{\text {мин }}}^{\infty} \tau \varphi(\tau) d \tau
$$


Интегрирование ура́внения (6) с учетом (4) и (5) дает

$$
\tau_{\mathrm{cp}}=\tau_{\text {мин }}+\frac{1}{k} \quad \text { или } \quad k=\frac{1}{\tau_{\mathrm{cp}}-\tau_{\text {мв }}} .
$$

\section{Поэтому окончательно получаем}

$$
\varphi(t)=\frac{1}{\tau_{\mathrm{cp}}-\tau_{\text {мин }}} \exp \left(-\frac{\tau-\tau_{\text {мин }}}{\tau_{\mathrm{cp}}-\tau_{\text {мин }}}\right)
$$

Зная закономерность протекания процесса (сушка, химическая реакция, горение) во времени, можно определить средний характерный параметр выходящего из реактора материала.

Например, зависимость относительного остатка горючего вещества $z$ от времени в области кинетического горения выражается в следующем виде:

$$
z=\exp (-A k \beta c \tau)
$$

где $A k$ - коэффициент, характеризующий кинетические свойства горения топлива; $\beta$ - стехиометрический коэффициент; $c$ - концентрация кислорода.

Средний относителыный остаток горючего вещества можно выразить каK

$$
\begin{gathered}
\bar{z}=\int_{\tau_{\text {мин }}}^{\infty} z(\tau) \varphi(\tau) d \tau \\
\text { или с учетом уравнений (7) и (8) } \\
\bar{z}=\frac{1}{\tau_{\text {ср }}-\tau_{\text {мин }}} \int_{\tau_{\text {мин }}}^{\infty} \exp \left(-\frac{\tau-\tau_{\text {мин }}}{\tau_{\mathrm{cD}}-\tau_{\text {мин }}}-A k \beta c \tau\right) .
\end{gathered}
$$

После интегрирования получаем

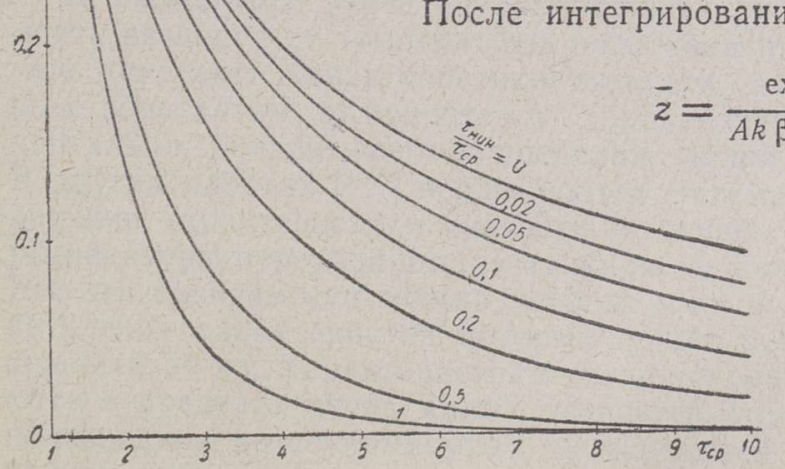

На рисунке изображена зависимость $\bar{z}=f\left(\tau_{\mathrm{cp}}\right)$ при $A k \beta c=1$ для раз. личных значений $\tau_{\text {мин }} / \tau_{\mathrm{cp}}$. Из графика видно, что минимально возможное Зависимость $z=f\left(\tau_{\mathrm{cp}}\right)$ при $A k \beta c=1$ для различвремя нахождения частиц в реакторе имеет достаточно большое значение. Следовательно, пренебрежение этим показателем может привести к значительным погрешностям. 


\section{Л И Т Е Р А Т Р А}

1. R e b o ux P., Phénomènes de fluidisation, Paris, 1954.

2. Федоров И. М., Теория и расчет процесса сушки во взвешенном состоянии, M., 1955.

3. П а леев И. И., Г у ре в ич М. А., Энергомашиностроение, № 3, 15-19 (1957).

4. Р ом а н ков.П. Г., Фролов В. Ф., Ж. прикл. химии, 35 , № $7,1526-1533$ (1962)

5. В а лх а р ж., Инж.-физ. ж., № 10, 33-39 (1963).

Институт термофизики и электрофизики Академии наук Эстонской.ССР
Поступила в редакцию 16/V 1966

R. $L O O N E$

\section{OSAKESTE KEEVKIHIS VIIBIMISE AJA EBAUHTLUSEST}

Artiklis on püütud matemaatiliselt väljendada keevkihis viibivate osakeste seesviibimise aja ebaühtluse mõju ja põhjendatud minimaalse ajaga $\tau_{\min }$ arvestamise vajalikkus.

R. $L O O N E$

\section{THE EFFECT OF NON-UNIFORM RESIDENCE TIME ON PARTICLES IN A FLUIDIZED BED}

The paper presents the results of an attempt to express mathematically the effect of non-uniform residence time on particles in a fluidized bed.. The author discusses the necessity of evaluating the minimum time $\tau_{\min }$. 